\title{
Robotic thymectomy for advanced thymic epithelial tumor: indications and technical aspects
}

\author{
Kwon Joong Na, Chang Hyun Kang \\ Department of Thoracic and Cardiovascular Surgery, Seoul National University Hospital, Seoul National University College of Medicine, Seoul, \\ Republic of Korea \\ Contributions: (I) Conception and design: All authors; (II) Administrative support: CH Kang; (III) Provision of study materials of patients: CH Kang; \\ (IV) Collection and assembly of data: All authors; (V) Data analysis and interpretation: All authors; (VI) Manuscript writing: All authors; (VII) Final \\ approval of manuscript: All authors. \\ Correspondence to: Chang Hyun Kang, MD, PhD. Department of Thoracic and Cardiovascular Surgery, Seoul National University Hospital, 101 \\ Daehak-ro, Jongno-gu, Seoul 03080, Republic of Korea. Email: chkang@snu.ac.kr.
}

\begin{abstract}
Robotic thymectomy is widely accepted as a valuable treatment option for surgical resection of thymic epithelial tumor as minimally invasive surgery has shown better early clinical outcomes than open surgery. Technical advances in robotic surgery have expanded the indications for robotic thymectomy, and the technique can be used to perform complete resection of advanced thymic epithelial tumor requiring concomitant resection of adjacent structures. To ensure complete resection, a multi-disciplinary approach, with thorough preoperative evaluation, must be adopted to determine whether a patient shows surgical indications for advanced thymic epithelial tumor. The early clinical outcomes after robotic thymectomy to treat advanced thymic epithelial tumor are promising; however, the long-term oncologic outcomes should be evaluated in the further studies.
\end{abstract}

Keywords: Thymoma; robotic surgical procedures; minimally invasive surgical procedures

Submitted Jun 27, 2019. Accepted for publication Sep 06, 2019.

doi: $10.21037 /$ jtd.2019.09.27

View this article at: http://dx.doi.org/10.21037/jtd.2019.09.27

\section{Introduction}

Thymic epithelial tumor (TET) is a rare neoplasm occurring in the anterior mediastinum. It is the most common primary neoplasm of the mediastinum in the adult population, and it frequently occurs alongside autoimmune diseases such as myasthenia gravis (1). Complete surgical resection is the primary treatment for TET (2) and many different types of surgical approaches have been introduced for thymectomy. Specifically, minimally invasive thymectomy (MIT) has several advantages over open thymectomy; for instance, it is associated with less trauma and fewer postoperative complications (3-8). However, many surgeons are still reluctant to adopt MIT, fearing incomplete resection or pleural seeding, particularly in cases of advanced-stage TET.

One form of MIT, robotic thymectomy, has been widely accepted as a valuable treatment option. Robotic surgical systems provide $3 \mathrm{D}$ visualization and can magnify the region of interest 10 times, and robotic arms are capable of free articulation. These advantages may be especially beneficial in patients with advanced TET invading the surrounding organs; such cases require complex resection or reconstruction, and are therefore technically demanding under thoracoscopy.

In the present paper, we describe our experience of robotic thymectomy to treat anterior mediastinal tumor, including the indications and surgical techniques of robotic thymectomy in patients with advanced TET.

\section{Indications for robotic thymectomy to treat advanced TET}

The important considerations for surgical resection of 
TET are complete resection (R0 resection). To prevent capsule disruption and tumor dissemination into the pleural space, which can lead to local recurrence of TET, it should be ensured that a surgeon should undertake only minimal tumor manipulation. Technical advancements in robotic surgery and its superior clinical outcomes over open thymectomy have expanded the surgical indications for this technique in the treatment of TET. However, to ensure complete resection in all cases of advanced TET, the indications for robotic thymectomy must be more strictly defined. A multi-disciplinary approach should be adopted, and thorough examination must be undertaken to ensure complete surgical resection of TET.

\section{Size and relationship with adjacent structures}

The size of the TET is critical in determining whether a robotic thymectomy should be performed. Large tumors are more often subject to excessive manipulation, increasing possibility of capsular disruption, as the anterior mediastinum is very narrow. Moreover, several studies have shown that TET size is an independent prognostic factor for overall and disease-free survival (9-11). Nonetheless, many centers have performed robotic thymectomy to treat large TET (3,12-14), and Kneuertz et al. (14) demonstrated that the procedure can be performed safely and effectively in a radical fashion. In our own center, malignant tumors larger than $8 \mathrm{~cm}$ are excluded from robotic thymectomy, while no size limit is applied to benign tumors (3), such as teratomas. In cases of invasive TET, free handling of the tumor is sometimes impossible because it risks capsule disruption and pleural seeding, so size criteria are more important in malignant tumor than benign tumors. In largesized tumor, intrathoracic space is also important because robotic arms cannot work efficiently without enough space. The bilateral lateral or subxiphoid approach are good alternatives in cases of large advanced TET.

Tumor infiltration into adjacent structures is also a major determinant for performing robotic thymectomy, and it should be carefully examined in preoperative imaging studies. Currently, TET invading the pericardium, phrenic nerve, lung, and innominate vein are not contraindications for robotic thymectomy in our center (3). Several previous studies in other centers have reported concomitant resection of these structures by robotic thymectomy (12-14). However, TET invasion of the chest wall or major vessels, including the superior vena cava, aorta, and main pulmonary artery, is considered a contraindication for robotic thymectomy, as the procedure is not technically feasible is such cases.

\section{Mediastinal lymph node metastasis}

Lymph node metastasis has been alleged as an independent prognostic factor in thymic malignancies, especially thymic carcinoma (15-17), so lymph node dissection plays a role in precise staging and improved prognosis prediction (15). In the past, we considered robotic thymectomy to be contraindicated when mediastinal lymph node dissection was necessary (3); however, we currently do not consider it as a contraindication because we can perform complete mediastinal lymph node dissection using the subxiphoid approach. More specifically, using the lateral approach, we could only remove the perithymic, internal mammary, hilar, and aortopulmonary lymph nodes. However, adopting the subxiphoid approach, we can additionally remove the paratracheal and lower cervical lymph nodes. Using the robotic subxiphoid approach, we can perform full and complete dissection of the anterior mediastinal lymph nodes.

\section{Pleural metastasis}

Robotic thymectomy in pleural metastasis can be performed in highly selected patients, however, in most of the cases, robotic thymectomy cannot be considered as surgical indication. Incidentally detected pleural nodule can be removed by robotic technique, however, thorough evaluation of the whole pleural space must be carried out. When pleural seeding is detected before surgery, open conversion due to multiple pleural seeding is quite common, so thoracoscopic exploration is a valuable option.

\section{Preoperative evaluation}

In patients with an anterior mediastinal mass suspected to be advanced TET, complete preoperative evaluation is needed, mainly to determine whether the mass is resectable or not. High-quality, contrast-enhanced thoracic computed tomography (CT) remains the mainstay preoperative imaging technique to determine whether complete resection is feasible (18-20). The CT scans can determine the size of the TET, evaluate the mass for signs of local invasion, and rule out metastatic disease (18-20). In particular, contrast-enhanced CT must be performed to examine the relationship between the adjacent major 


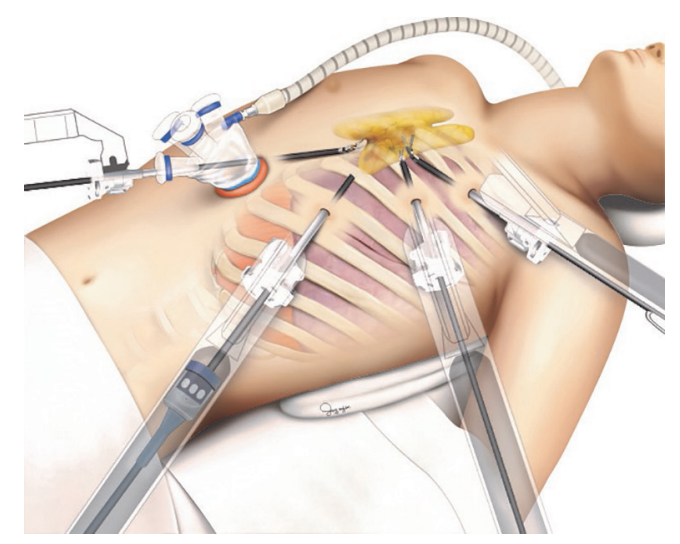

Figure 1 The modified subxiphoid robotic thymectomy.

vascular structures and TET. Usually, contrast-enhanced CT scan is sufficient to evaluate the resectability of TET, although chest magnetic resonance imaging is sometimes useful when TET invasion into the major vessels is not evidenced by CT (21). As advanced TET is more likely to show lymph node, pleural, or distant metastasis than earlystage TET, combined positron emission tomography/CT may be useful to determine whether intra- or extra-thoracic metastasis is present. Pulmonary function tests must also be performed, and the results should be examined carefully in patients with advanced TET, as the concomitant resection of the lung may be necessary.

\section{Approach}

In our center, we previously performed robotic thymectomy by lateral thoracic approach as we reported our initial experience of robotic thymectomy $(3,4)$. However, we currently changed our strategy to subxiphoid approach for robotic thymectomy since we initiated first subxiphoid robotic thymectomy in 2016. For those patients who are suspicious for advanced TET requiring hilar structures dissection for complete resection, we use a novel technique called "modified subxiphoid approach".

\section{Lateral thoracic approach}

Most studies reporting the outcomes of robotic thymectomy have used the lateral thoracic approach for surgical resection of TET $(3,4,12-14)$. This approach is usually performed using the three-arm technique. Resection of adjacent structures using this approach has also been reported, including the pericardium, phrenic nerve, innominate vein, and lung. In a report by Kneuertz et al. (14), $50 \%$ of patients who were subjected to robotic thymectomy for large TET resection had undergone concomitant resection of adjacent structures. They concluded that concomitant resection of the pericardium, lung, and phrenic nerve was technically feasible using the lateral thoracic approach, and only two of their patients required thoracotomy for additional access to ensure safe resection. We have reported our own experience of robotic thymectomy using the lateral thoracic approach $(3,4)$.

\section{Subxiphoid approach}

Recently, several authors reported the safety and technical feasibility of subxiphoid robotic thymectomy for surgical resection of TET $(22,23)$. This approach provides excellent visualization of the mediastinum, particularly both upper poles of the thymus and the bilateral phrenic nerves, and it allows safe and effective dissection of the mediastinal fatty tissue. Although subxiphoid robotic thymectomy is usually performed using the three-arm technique $(22,23)$, we mostly use the four-arm technique in cases of advanced TET to ensure safe handling of the thymic tissues and allow complex procedures to be performed. Suda et al. reported the technical feasibility of subxiphoid robotic thymectomy, also mentioning that concomitant resection of lung and pericardium is possible using articulating robotic instruments such as the robotic stapler (23). Moreover, a recent paper by Zhang et al. demonstrated good early clinical outcomes of subxiphoid robotic thymectomy (24).

\section{Modified subxiphoid approach}

In our center, we recently developed a novel technique, named the "modified subxiphoid approach" for complete resection of advanced TET. Briefly, the patient is placed in a position similar to that adopted in the lateral thoracic approach (supine position with ipsilateral side elevation). A vertical incision is made in the subxiphoid area, followed by three additional robotic ports on the hemithorax, where the mass is located (Figure 1). This approach has several advantages: (I) the surgical view of the thymic tumor and pulmonary hilum is better than with the conventional subxiphoid approach, allowing a more complex operation to be performed around the hilum, including combined pulmonary lobectomy; (II) conflict between the sternum and robotic camera can be minimized; (III) the lower pleural space can be better visualized. 


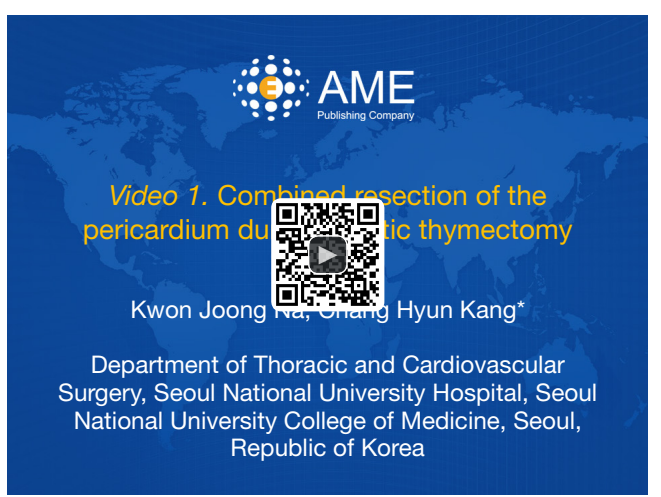

Figure 2 Combined resection of the pericardium during robotic thymectomy (25).

Available online: http://www.asvide.com/watch/33087

\section{Combined resection of adjacent structures}

\section{Pericardium (Figure 2)}

The pericardium is one of the most common structures requiring concomitant resection in cases of advanced TET. If surgeons suspect direct invasion by TET, the pericardium should be opened, with sufficient margin from the TET, and a concomitant resection can be performed using monopolar scissors, a harmonic scalpel (Ethicon Endosurgery, Cincinnati, OH, USA), or da Vinci vessel sealers (Intuitive Surgical Inc., Mountain View, CA, USA). The most challenging procedures while performing concomitant resection of the pericardium are when there is dense adhesion between the pericardium and the major vessels. Even when the TET shows no direct infiltration into the major vessels, there is sometimes dense adhesion inside the invaded pericardium. As robotic surgical systems provide a $3 \mathrm{D}$ view with $10 \times$ magnification, and surgeons can use four robotic arms to carry out precise procedures, with assistance from articulating arms, fine dissection can be performed between major vessels and densely adherent thymic tissue.

In our center, we always reconstruct pericardial defects using a prosthetic membrane to prevent severe adhesion between cardiac structures and the lower border of the sternum. We use an articulating needle driver, anchoring the sheet to the surrounding pericardium with multiple interrupted sutures using silk or polypropylene sutures.

\section{Phrenic nerve}

Both phrenic nerves are surrounded by the thymic tissue, so they are also commonly involved in advanced TET.
Ipsilateral hemidiaphragm elevation in patients with TET suggests direct invasion and paralysis of the phrenic nerve; the concomitant resection of the phrenic nerve is necessary in those patients. However, many other patients who require this procedure do not present any preoperative signs or symptoms of diaphragmatic paralysis. As the course of the phrenic nerve is not visualized under imaging studies, involvement of the phrenic nerve in TET is often identified during surgery itself.

When the phrenic nerve abuts the TET without evidence of invasion or encasement of the nerve, meticulous dissection between the phrenic nerve and the TET can usually be achieved without difficulty using robotic surgical techniques. If the direct invasion or encasement of the phrenic nerve by the TET is observed, concomitant resection of the phrenic nerve segment is necessary to ensure complete resection of the TET. In our center, we mostly perform diaphragmatic plication for the diaphragm paralysis, followed by the concomitant resection of the TET and phrenic nerve. Diaphragmatic plication is performed using additional thoracoscopic technique after complete resection of the TET by robotic thymectomy. However, we do not perform diaphragmatic plication in patients who have undergone concomitant resection of a large volume of lung parenchyma. Some studies have reported successful immediate reconstruction of the phrenic nerve in cases of phrenic nerve injury during mediastinal tumor resection (26,27). Most relevant studies have reported using sural nerve transfer to reconstruct the phrenic nerve, with favorable outcomes for diaphragmatic function $(26,27)$. Recently, we performed a reconstruction after a short, segmental resection of the phrenic nerve using end-to-end anastomosis by robotic surgery. We used $5 / 0$ polypropylene suture for the end-to-end anastomosis, and some patients showed restored diaphragmatic function by fluoroscopy during follow-up (Figure 3).

\section{Lung}

Many cases require wedge resection of the lung to ensure complete TET resection; this can be performed using the EndoWrist stapler (Intuitive Surgical Inc.) —an articulated stapler that is attached to the da Vinci system (Intuitive Surgical, Inc.), allowing the surgeon to carry out resection with control and autonomy from the surgery console. By inserting the stapler through subxiphoid or lateral thoracic ports, wedge resection of the lung can be performed.

In some cases, lobectomy of the lung is required to 


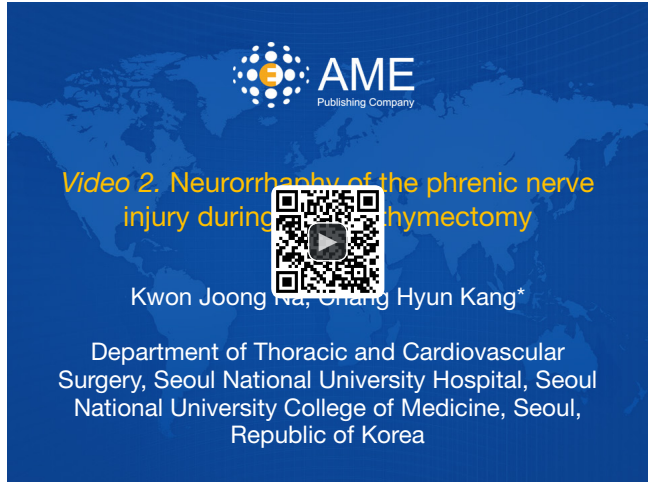

Figure 3 Neurorrhaphy of the phrenic nerve injury during robotic thymectomy (28).

Available online: http://www.asvide.com/watch/33088

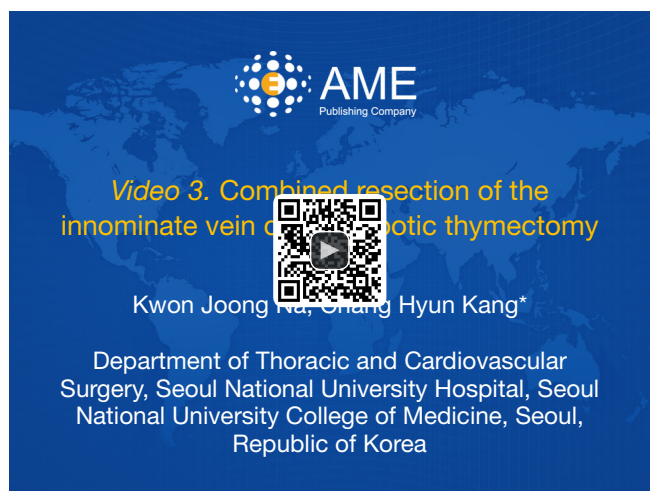

Figure 4 Combined resection of the innominate vein during robotic thymectomy (31).

Available online: http://www.asvide.com/watch/33089

ensure complete resection of the TET, particularly when the tumor is infiltrating close to the hilar structures or broadly across the surface of the lung. To date, no studies have reported concomitant resection of TET with major pulmonary resection by robotic surgery. In the past, we preferred to use additional thoracoscopic technique if the patient required lobectomy of the lung to ensure complete TET resection (3). As described earlier, we now prefer the modified subxiphoid approach for this purpose, and concomitant major pulmonary resection plus TET resection is technically feasible and can be safely performed. Some previous studies have reported that thoracoscopic lobectomy through the subxiphoid incision is technically feasible and safe $(29,30)$, and that it could be a good surgical option in patients who have undergone subxiphoid robotic thymectomy and require combined major pulmonary resection to ensure complete TET resection.

\section{Innominate vein (Figure 4)}

When TET infiltrates the innominate vein, concomitant resection can be performed using a robotic stapler. To perform this procedure safely, the upper cervical poles and both the proximal and distal end of the innominate vein must be completely dissected. Distal end invasion is not problematic in most cases: even when the tumor invades the left mammary vein, the vein can be resected at the level of proximal left jugular vein or subclavian vein. However, if the tumor invades the proximal end of the innominate vein, the possibility of superior vena cava invasion must be considered. Partial resection of the superior vena cava can be performed if technically feasible. However, considering postoperative narrowing of the superior vena cava and risk of major bleeding, open conversion may be a safer approach.

\section{Postoperative management}

A drain should be placed across the mediastinum to drain both thoracic cavities. The Jackson Pratt drain is usually adequate; however, a chest tube should be placed in patients who have undergone concomitant resection of the lung parenchyma. Respiratory symptoms or signs should be carefully monitored in patients who have undergone concomitant lung or phrenic nerve resection. Patients are usually discharged 2-3 days after surgery if they recover without complications.

\section{Outcomes of robotic thymectomy}

Between May 2008 and June 2019, 291 patients underwent robotic thymectomy to treat treatment anterior mediastinal tumors in our institute, 168 of whom had a TET, and their clinical profiles are presented in Table 1 .

No postoperative mortality occurred, while seven patients showed postoperative complications (4.2\%). Major complications with a Clavidien-Dindo classification of more than grade 3 occurred in one patient $(0.6 \%)$. The mean hospital stay after surgery was $3.1 \pm 2.5$ days (range, $1-20$ days).

Forty-four patients with large tumors $(>6 \mathrm{~cm})$ underwent robotic thymectomy. Only one open conversion was necessary because of possible invasion into the superior vena cava. Despite their large size, most of these tumors were at an early stage: only one was stage III and two were stage 
Table 1 Clinical profiles of patients who underwent robotic thymectomy to treat thymic epithelial tumor

\begin{tabular}{lc}
\hline Variables & Number $(\%)(\mathrm{n}=168)$ \\
\hline Sex (male) & $83(49.4)$ \\
Age (years) & $55.7 \pm 11.8$ \\
Cell types & \\
A & $14(8.3)$ \\
AB & $56(33.3)$ \\
B1 & $38(22.6)$ \\
B2 & $14(8.3)$ \\
B3 & $13(7.7)$ \\
Thymic carcinoma & $28(16.7)$ \\
Other types & $2(1.2)$ \\
Stages & \\
I & $152(90.5)$ \\
II & $3(1.8)$ \\
III & $7(4.2)$ \\
IV & $6(3.6)$ \\
\hline
\end{tabular}

IV tumors, indicating that invasiveness does not always correspond with TET size. Recurrence was noted in two patients: one was cured by reoperation and the other is still alive with the disease following chemoradiation therapy.

A total of 29 patients $(17.3 \%)$ underwent concomitant resection of adjacent organs. Lung resection was performed in 20 patients (including four lobectomies), pericardium resection in 18 patients, phrenic nerve resection in six patients, and innominate vein resection in six patients. Two patients who underwent phrenic nerve resection received neurorrhaphy. Lymph node sampling or dissection was performed in 41 patients (24.4\%), although full mediastinal lymph node dissection according to the AJCC lymph node map was performed in only seven patients $(4.2 \%)$. Lymph node metastasis was confirmed in four patients (2.4\%).

One patient died of intestinal lymphoma not related to the TET. The 7-year overall survival rate of robotic thymectomy to treat TET was $98 \%$ in our institute. Recurrence was noted in two patients with thymoma and three with thymic carcinoma. Three of these had stage IV TET before surgery. Pleural seeding was a common mechanism of recurrence in patients with thymoma, while multi-organ recurrence was common in thymic carcinoma.

\section{Summary}

As the early clinical outcomes of MIT are superior to those of open thymectomy, robotic thymectomy has gained in popularity over the decades. Technical advancements in robotic surgical systems have allowed complex procedures to be performed in adjacent organs, enabling complete resection of advanced TET using robotic surgery. However, the surgical indications in patients with advanced TET must be subject to a multi-disciplinary approach and thorough preoperative evaluation, because complete resection without tumor disruption is critical in the surgical treatment of TET. Furthermore, the long-term oncological outcomes after robotic thymectomy should be evaluated in patients with advanced TET.

\section{Acknowledgments}

None.

\section{Footnote}

Conflicts of Interest: The authors have no conflicts of interest to declare.

Ethical Statement: The authors are accountable for all aspects of the work in ensuring that questions related to the accuracy or integrity of any part of the work are appropriately investigated and resolved.

\section{References}

1. Mao ZF, Mo XA, Qin C, et al. Incidence of thymoma in myasthenia gravis: a systematic review. J Clin Neurol 2012;8:161-9.

2. Girard N, Ruffini E, Marx A, et al. Thymic epithelial tumours: ESMO Clinical Practice Guidelines for diagnosis, treatment and follow-up. Ann Oncol 2015;26:v40-55.

3. Kang CH, Hwang Y, Lee HJ, et al. Robotic thymectomy in anterior mediastinal mass: propensity score matching study with transsternal thymectomy. Ann Thorac Surg 2016;102:895-901.

4. Seong YW, Kang CH, Choi JW, et al. Early clinical outcomes of robot-assisted surgery for anterior mediastinal mass: its superiority over a conventional sternotomy approach evaluated by propensity score matching. Eur J Cardiothorac Surg 2014;45:e68-73.

5. Jurado J, Javidfar J, Newmark A, et al. Minimally invasive 
thymectomy and open thymectomy: outcome analysis of 263 patients. Ann Thorac Surg 2012;94:974-81; discussion 981-2.

6. Cheng YJ, Kao EL, Chou SH. Videothoracoscopic resection of stage II thymoma: prospective comparison of the results between thoracoscopy and open methods. Chest 2005;128:3010-2.

7. Meyer DM, Herbert MA, Sobhani NC, et al. Comparative clinical outcomes of thymectomy for myasthenia gravis performed by extended transsternal and minimally invasive approaches. Ann Thorac Surg 2009;87:385-90; discussion 390-1.

8. Toker A, Eroglu O, Ziyade S, et al. Comparison of early postoperative results of thymectomy: partial sternotomy vs. videothoracoscopy. Thorac Cardiovasc Surg 2005;53:110-3.

9. Roden AC, Eunhee SY, Jenkins SM, et al. Modified Masaoka stage and size are independent prognostic predictors in thymoma and modified Masaoka stage is superior to histopathologic classifications. J Thorac Oncol 2015;10:691-700.

10. Okumura M, Yoshino I, Yano M, et al. Tumour size determines both recurrence-free survival and diseasespecific survival after surgical treatment for thymoma. Eur J Cardiothorac Surg 2019;56:174-81.

11. Fukui T, Fukumoto K, Okasaka T, et al. Prognostic impact of tumour size in completely resected thymic epithelial tumours. Eur J Cardiothorac Surg 2016;50:1068-74.

12. Keijzers M, Dingemans AM, Blaauwgeers H, et al. 8 Years' experience with robotic thymectomy for thymomas. Surg Endosc 2014;28:1202-8.

13. Marulli G, Maessen J, Melfi F, et al. Multi-institutional European experience of robotic thymectomy for thymoma. Ann Cardiothorac Surg 2016;5:18-25.

14. Kneuertz PJ, Kamel MK, Stiles BM, et al. Robotic thymectomy is feasible for large thymomas: a propensitymatched comparison. Ann Thorac Surg 2017;104:1673-8.

15. Hwang Y, Kang CH, Park S, et al. Impact of Lymph Node Dissection on Thymic Malignancies: Multi-Institutional Propensity Score Matched Analysis. J Thorac Oncol 2018;13:1949-57.

16. Hwang Y, Park IK, Park S, et al. Lymph node dissection in thymic malignancies: implication of the ITMIG lymph node map, TNM stage classification, and recommendations. J Thorac Oncol 2016;11:108-14.

17. Park IK, Kim YT, Jeon JH, et al. Importance of lymph node dissection in thymic carcinoma. Ann Thorac Surg 2013;96:1025-32.

18. White DB, Hora MJ, Jenkins SM, et al. Efficacy of chest computed tomography prediction of the pathological TNM stage of thymic epithelial tumours. Eur J Cardiothorac Surg 2019. [Epub ahead of print].
19. Moon JW, Lee KS, Shin MH, et al. Thymic epithelial tumors: prognostic determinants among clinical, histopathologic, and computed tomography findings. Ann Thorac Surg 2015;99:462-70.

20. Shen Y, Gu Z, Ye J, et al. CT staging and preoperative assessment of resectability for thymic epithelial tumors. J Thorac Dis 2016;8:646.

21. Ried M, Hnevkovsky S, Neu R, et al. Impact of Surgical Evaluation of Additional Cine Magnetic Resonance Imaging for Advanced Thymoma with Infiltration of Adjacent Structures: The Thoracic Surgeon's View. Thorac Cardiovasc Surg 2017;65:244-9.

22. Suda T. Subxiphoid thymectomy: single-port, dual-port, and robot-assisted. J Vis Surg 2017;3:75.

23. Suda T, Kaneda S, Hachimaru A, et al. Thymectomy via a subxiphoid approach: single-port and robot-assisted. J Thorac Dis 2016;8:S265-71.

24. Zhang H, Chen L, Zheng Y, et al. Robot-assisted thymectomy via subxiphoid approach: technical details and early outcomes. J Thorac Dis 2018;10:1677-82.

25. Na KJ, Kang CH. Combined resection of the pericardium during robotic thymectomy. Asvide 2020;7:047. Available online: http://www.asvide.com/watch/33087

26. Schoeller T, Öhlbauer M, Wechselberger G, et al. Successful immediate phrenic nerve reconstruction during mediastinal tumor resection. J Thorac Cardiovasc Surg 2001;122:1235-7.

27. Kim TY, Hong KW, Kim KI, et al. Phrenic nerve reconstruction during anterior mediastinal tumor resection. Korean J Thorac Cardiovasc Surg 2002;35:560-3.

28. Na KJ, Kang CH. Neurorrhaphy of the phrenic nerve injury during robotic thymectomy. Asvide 2020;7:048. Available online: http://www.asvide.com/watch/33088

29. Gonzalez-Rivas D, Lirio F, Sesma J, et al. Subxiphoid complex uniportal video-assisted major pulmonary resections. J Vis Surg 2017;3:93.

30. Hernandez-Arenas LA, Lin L, Yang Y, et al. Initial experience in uniportal subxiphoid video-assisted thoracoscopic surgery for major lung resections. Eur J Cardiothorac Surg 2016;50:1060-6.

31. Na KJ, Kang CH. Combined resection of the innominate vein during robotic thymectomy. Asvide 2020;7:049. Available online: http://www.asvide.com/watch/33089

Cite this article as: $\mathrm{Na} \mathrm{KJ}$, Kang $\mathrm{CH}$. Robotic thymectomy for advanced thymic epithelial tumor: indications and technical aspects. J Thorac Dis 2020;12(2):63-69. doi: 10.21037/jtd.2019.09.27 